\title{
26. \\ Enterprise Integration Approaches in Healthcare: A Decade of Trial and Error
}

\author{
V. Jagannathan \\ West Virginia University \& MedQuist, Inc. \\ juggy@csee.wvu.edu
}

This paper chronicles the different approaches for enterprise integration used in the field of healthcare over the past decade, and which approach succeeded and which failed. It ends with the new approach just launched through the Health Level 7 standards organization with support from the Health and Human Services in US.

\section{INTRODUCTION}

Integration and interoperability issues are not new to healthcare. They are the very reason why large standards body such as Health Level 7 (HL7) and EU CEN TC 251 exists with the goal of simplifying integration and promoting interoperability. The problem of interoperability however takes on massive connotations in healthcare, unlike most other disciplines. One needs to look no further than the National Healthcare Information Infrastructure (NHII) goals in US and similar efforts around the world to understand what is at stake. Very simply stated, the goals of these initiatives is to provide access to any pertinent information which furthers the treatment of any individual at any location and point in time by any authorized care giver. Now if one overlays the scope of information of individuals - also dubbed their longitudinal electronic health record - which tracks what was done to individuals from cradle to grave, and any relevant medical treatment information the scope becomes a bit more clearer. Now, if we extrapolate to hundreds of millions to billions of the general populace, privacy and confidentiality considerations, and the tens of thousands of information systems that are deployed in the real world, it is clear that this is a problem that is not going to be solved in a hurry. This document chronicles the different approaches tried to date and the new approach being attempted by the NHII initiative to address interoperability and integration in healthcare.

\section{APPROACHES}

\subsection{Classification of approaches}

Standards clearly play a part when diverse systems need to be integrated. One of the traditional approaches to integration, which has been very successful in financial communities, is the Electronic Data Interchange (EDI) standards. In healthcare, this approach is facilitated by Health Level 7 (HL7) standard. The HL7 standard is primarily a message-oriented approach to integration (Hinchley, 2003). 
The latter part of last decade, gave credence to another approach that can be best described as "Service Oriented Architectures". Proponents of this approach include the Object Management Group and their Healthcare Task force (HDTF, 2004).

Another approach, which at the time it was proposed was unique to healthcare, is the notion of "Visual Integration". Here, the integration happens at the customer/client user end of the applications (CCOW, 2004).

Lastly, but not the least, there are approaches which hinge around ontology and agent-based architectures and peer-to-peer communication protocols. The impact of these types of architectures is limited in healthcare to date though gaining in ground given the focus on evidence-based practice of medicine (AgentCities, 2002).

All of the above approaches and their strengths and weaknesses are highlighted in the next section.

\subsection{Message-Oriented Integration}

HL7 is a well established standard and is the primary mechanism by which most applications in healthcare communicate with each other. HL7 has a very rigorous approach to creating message structures that are based on a general purpose Reference Information Model of healthcare that supports variety of workflows in healthcare (Hinchley, 2003). For instance, there are specific messages one can use to communicate that a patient has been admitted, discharged or transferred. Hundreds of message definitions exist and they serve the purpose of communicating between various applications in healthcare.

The biggest advantage with this approach in healthcare is that it is well established. However, since every system behaves like a complete application and stores whatever information it needs, there tends to be significant duplication of information. Almost every clinical application deployed in a hospital for instance, needs to have some patient demographic information. This is typically acquired during the hospital registration process. What is typically done is this information is communicated individually in a separate message to every application that needs this information - typically fifteen to twenty applications in just one hospital. Every one of these system will store this common information - leading to significant duplication of some common information. This approach has given rise to an entire category of applications called "Interface Engines" whose sole purpose in life is to make the process of getting the sending and receiving applications to handle the vagaries of the messages that are being communicated.

A variation of this approach that was attempted in healthcare, include one where the messages, instead of being an ASCII string, was instead an object with rich structure. Sending and receiving messages as objects save the sending and receiving applications from having to parse through the syntactic structure of the message. It made the process a bit more efficient at run time at the expense of the complexity of the standard itself. Andover Working Group and ActiveX for Healthcare and HL7 Special Interest Group on Object Brokering Technologies (SIGOBT) promoted this approach in 1998-2000 time frames (Jagannathan et al, 1998). This particular effort failed in its entirety and was abandoned. The reason this failed was because there was not much perceived value for "object version of messages", as most vendors had already invested in parsing HL7 message standards and they were unwilling to replace something that worked really well. 
Another standard which supports this paradigm and well established in healthcare is the Digital Imaging and Communication in Medicine (DICOM) standard. This standard is used to communicate digital images such as XRays, MRIs, CT Scans etc and enjoys uniform support and conformance from most imaging solution vendors (DICOM, 2004).

In message-oriented approaches there is an implicit assumption that information is being sought only in the context of ONE application and the information that particular application provides is all that will be accessible to the user. It is simply untenable to assume that there is going to one application that warehouses all patient information from cradle to grave for all patients. Purely message oriented approaches to integration will not satisfy the NHII goals.

\subsection{Service-Oriented Architectures}

An alternative approach is the one advocated by the Object Management Group $(\mathrm{OMG})$ and others. The notion here is simply that each application provides a programmatic interface to which other applications can send messages and get back responses. A request-response paradigm for integration is dubbed as "ServiceOriented Architectures" (SOA). OMG's Healthcare Task Force between 1996 and 2002 , came up with a series of specifications in very specific healthcare application domains that support this model.

OMG's Common Object Request Broker Architecture (CORBA) provided an interoperable framework that allowed clients and servers running on different platforms and different language bindings to interoperate. This was and still is a mature technology and it had proved itself in numerous other domains such as telecom and finance. It also separates specifications into horizontal and vertical segments. Horizontal specifications are those that are applicable to all industries and include infrastructure pieces such as security and event notification and communication. Vertical specifications are industry specific.

Though some of the specifications developed by the Healthcare Domain Task Force (DTF) (HDTF, 2004) enjoyed some adoption, the OMG effort in healthcare failed for a number of reasons. These include: 1) A perception that this approach of using CORBA competed with the use of DCOM and Microsoft technologies, 2) The level of complexity and the bar for entry was significant for application developers in healthcare, and 3) This effort competed with HL7 charter for providing interoperability standards and specification in healthcare. HL7 had much broader representation than the technically oriented OMG.

The European Committee for Standardization CEN, Technical Committee 251 is focused on healthcare informatics ${ }^{76}$. At various times they have investigated SOA architectures and currently the WG1 is focused on specifying such solutions as part of their "Healthcare Common Information Services (HCiS) effort.

Though the OMG effort failed, there is a resurgence of SOA, due to the advancement in XML-related technologies in general and Web Services in particular. This aspect of integration is discussed in section 3.0 below. It is also implicitly assumed that to realize the goals of NHII a combination of SOA and message oriented solutions will need to be deployed. And it is quite conceivable that

\footnotetext{
${ }^{76}$ European Union - CEN TC 251 - Healthcare Informatics Technical Committee. http://www.centc251.org
} 
the specifications developed by OMG might re-appear in a morphed form to address the goals of the EHR and NHII (See section 4.0).

\subsection{Visual Integration}

One unique approach to integration that has taken some hold is a focus on providing value to the users by visually integrating the client applications. The approach here, embodied in a standard named Clinical Context Object Workgroup (CCOW) is to take the client side of multiple applications and get each of them to switch context in tandem to user actions. The following time-sequence diagram and analysis explains this concept in greater detail. In the following, we assume that there are two vendor applications - labeled "App A" and "App B", one user, labeled "User P" and a context management application labeled as "CM". The numbered items are interaction between applications and user and various applications.

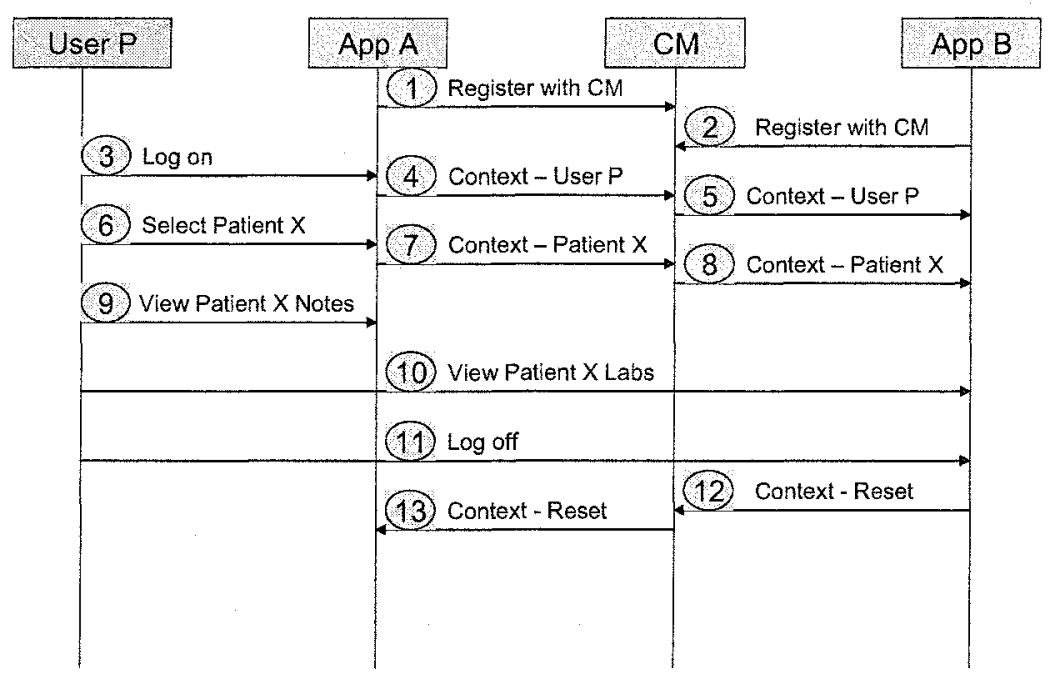

1. Application A registers with the context manager (CM). This allows $\mathrm{CM}$ to notify application A when it needs to.

2. Application B registers with the context manager (CM). This allows $\mathrm{CM}$ to notify application $B$ when it needs to.

3. User P logs on to Application A.

4. Application A sends a notification to $\mathrm{CM}$ stating the user context is $\mathrm{P}$ essentially indicating that $\mathrm{P}$ has logged on.

5. CM propagates (notifies) the context to Application B. Now application B knows user $\mathrm{P}$ is logged on and resets the context of the application to reflect that.

6. User $P$ interacts with application $A$ and selects patient $X$.

7. Application A sends this contextual information to $C M$.

8. CM propagates that Patient $X$ is the context to Application B. 
9. User $\mathbf{P}$ interacts with Application $\mathbf{A}$ and reviews say Patient $\mathbf{X}$ clinical notes.

10. User $P$ interacts with Application $B$ and reviews the lab results for patient $X$. Note that the user did not have to $\log$ on to Application B nor select Patient $\mathrm{X}$. Application $\mathrm{B}$ already knows the context by receiving context information from CM.

11. User P logs off on Application B.

12. Application $B$ sends notification to $\mathrm{CM}$ to reset the context - equivalent to not having context or nobody is logged on.

13. CM propagates this to Application A and hence Application A logs the user off and resets the context. By virtue of logging off on Application B, user is logged off from Application A as well.

Application A and B in above are referred to as Context Participants (CP) in the CCOW standard. CCOW standard enjoys modest support in healthcare. However, when it is used for purely providing a single-sign-on capability between multiple applications, there are more general and simpler approaches available. The simplest of these approaches include solutions provided by password management applications. Here a browser keeps track of (caches) username/password pairs for multiple web-based applications and simply use them to $\log$ on to multiple applications. Other efforts to provide single sign on solutions include efforts within the Oasis group such as Secure Access Markup Language (SAML, 2004).

\subsection{Agent and Ontology-based Architectures}

Agent-based and artificial intelligence based techniques have been around for a while. They gained major significance in late 80's with the advent of fifth generation computing in Japan. However, the technologies failed to live up to the expectations of the users and the techniques promoted took a back seat to the evolution of internet and web in the $90 \mathrm{~s}$. There is currently a resurgence of interest in knowledge and agent-based solutions. Agentcities.com is a group focused on promoting agent technologies. A subgroup of this group, work on healthcare applications (AgentCities, 2002) with hopes of developing and promoting solutions for integration using multi-agent architectures.

Part of the basis for this effort is knowledge ontology of health care concepts. The "Protége" project at Stanford provides a tool that will allow users to create their own ontology ( $\mathrm{Li}$ et al, 2000). It has been used extensively by a number of different groups to develop ontologies in healthcare. Another related effort in the tools arena is the "OpenGalen" project. This project seeks to build an open framework for supporting a terminological server that will allow documentation using standard terminologies ${ }^{77}$.

Medical vocabularies are complex and hitherto have lead to a number of different standards with varying specificity and goals. The International Classification of Diseases (ICD), developed by the World Health Organization is a widely used classification scheme. ICD 9, the 9th version, is used in US for billing and reimbursement purposes. ICD 10 is a better classification of diseases and is yet to find widespread adoption in US. Current Procedural Terminology (CPT) developed by the American Medical Association, is a classification of procedures

\footnotetext{
${ }^{77}$ OpenGalen Foundation, Nijmegen, the Netherlands (http://www.opengalen.org/index.html)
} 
performed by physicians and is used very heavily in US to pay for physician services. ICD and CPT and various variations of it have as their primary goal of supporting the reimbursement process (Buck, 2002) and have less relevance for actually providing care. In order to support the goal of reducing medical errors and providing evidence-based decision support at the point of service, other vocabularies and coding schemes are more relevant. The Unified Medical Language System (UMLS) developed by the National Library of Medicine (NLM) is a scheme to allow the classification and indexing of medical knowledge (UMLS, 2004). The Systematized Nomenclature of Medicine: Clinical Terms (SNOMED CT) is a systematic classification of medical terms and shows the promise of representing medical conditions and treatments with aim towards supporting clinical decision support. SNOMED is now made available freely in US as part of the NHII promotion effort ${ }^{78}$. Ontology and knowledge driven integration of healthcare applications is a necessity if the goals of safety and quality in healthcare delivery has to be realized.

\section{IMPACT OF XML TECHNOLOGIES ON HEALTHCARE INTEGRATION}

\subsection{Special Interest Group in XML in HL7}

In 1997, HL7 established a Special Interest Group (SIG) on SGML - the precursor to HTML and XML. This group recognized early on that a mark-up language will simplify encoding clinical content. When XML standard was established, this SIG switched their efforts to XML. The lobbying effort by this group resulted in the wholesale adoption of XML as the core technology to be used for specifying all new standards specification from HL7. The Reference Information Model (RIM) which uses Unified Modelling Language (UML) for its core representation, supports translating actual message definitions into XML. One of the core specifications that were adopted early on was encoding of clinical content using XML. This specification was called Clinical Document Architecture or CDA for short. Currently, the second generation of these specifications are in the works. The CDA was a departure from the usual types of specifications that HL7 supported. The CDA was not designed as a message - but rather as a free standing content document. It could be delivered as a payload in a message or it could be delivered as a response to a request. How the content is to be used and delivered was left to the implementers of systems (CDA, 2004).

\section{$3.2 \mathrm{ebXML}$ and Healthcare}

Electronic Business XML (ebXML) has traction particularly in Europe and the primary goal of this effort is to enable electronic transactions between business partners. This standard is supported by the Oasis group (ebXML, 2001) and (Kotok and Webber, 2002). This standard is being explored by Centers for Disease Control (CDC) and Federal Drug Agency (FDA) to allow organizations to report to them on communicable and syndromatic disease outbreaks and drug-drug interactions.

\footnotetext{
${ }^{78}$ Systematized Nomenclature of Medicine Clinical Terms (SNOMED CT) : http://www.snomed.org
} 


\subsection{Integration Demonstrations}

HL7 orchestrated a sequence of demonstration using XML technologies starting in 1999. These demonstrations progressively became more and more complex over the years. In 2004, the demonstrations joined hands with a number of other organizations such as HIMSS and RSNA to put together what was dubbed the "Integrated Healthcare Enterprise (IHE)" "79. IHE actually started out as a demonstration of integration technology focused in the radiology domain in 2001. The IHE demonstration this year (2004) combined all the elements discussed in this paper to this point - HL7 messaging, DICOM standards, CCOW standards, CDA standards and use of XML-web services to access CDA documents. Clearly the healthcare pendulum has swung in the direction of using XML and web services. Nearly 25 different vendors and numerous standards group participated in this demonstration showcasing that fairly significant interoperability is indeed feasible. However, these efforts are nowhere close to supporting the true goal of NHII - that of a longitudinal electronic health record. This leads logically to the next effort discussed in the next section.

\section{FUNCTIONAL MODEL OF ELECTRONIC HEALTH RECORD - DRAFT STANDARD FOR TRIAL USE (DSTU)}

\subsection{NHII Imperatives}

The Institute of Medicine came up with a series of reports which raised the national conscience on the state of healthcare. Their report, "To err is human" (Institute of Medicine, 2001) showed that there were 98,000 preventable errors in healthcare annually in US. A follow on report, showed that serious quality concerns persist in healthcare (Institute of Medicine, 2001b). To address all of these, the National Healthcare Information Infrastructure (NHII) initiative was born. NHII is an initiative that is currently managed by the Department of Health and Human Services in US. NHII is " . . the set of technologies, standards, applications, systems, values, and laws that support all facets of individual health, health care, and public health" (NCVHS, 2000)

\subsection{EHR - Draft Standard for Trial Use}

Institute of Medicine under the direction of Health and Human Services (HHS) released a new report in summer 2003 titled: "Key Capabilities of an Electronic Health Record System" (Institute of Medicine, 2003). This report became the seed for a fast-track effort at HL7 to define a functional model for what constitutes an EHR - driven by HHS. The goal that HHS has is to provide financial incentives through their CMS (Medicare/Medicaid) wing to further adoption and implementation of EHR starting in 2004. In order for them to provide such an incentive, there needs to be a definition in place what constitutes an EHR and what functionality it needs to support. This is what HL7 is currently trying to do. An initial effort which resulted in a functional model with over 1000 elements was rejected by the HL7 membership in September 2003 as being overly complex. A

\footnotetext{
${ }^{79}$ Integrating Healthcare Enterprise (IHE): http://www.rsna.org/IHE/index.shtml.

${ }^{80}$ National Healthcare Information Infrastructure (NHII): http://aspe.hhs.gov/sp/nhii/
} 
revamped functional model was balloted again and has now been adopted as EHR Functional Model - Draft Standard for Trial Use in July 2004 (HL7, 2004).

The revamped model summarizes the functionality of the EHR in three categories: 1) Direct Care, 2) Supportive functions and 3) Infrastructure. Direct care functions include applications that support capturing orders, medication and clinical documentation. Supportive functions include providing decision support, alerts, drug-drug interaction warnings etc. Infrastructure functions include addressing security and privacy concerns, Public-Key Private-Key infrastructure and the like.

The functional model is a blue print for what EHR functionality needs to be supported. The specification and mapping of specific standards on how this functionality needs to be supported so there can be a broader integration and interoperability is a future task.

\section{CONCLUSIONS}

This paper chronicles the efforts to develop interoperable solutions in healthcare over the past decade. The current status is also presented. The problem of enterprise integration and interoperability has been around for a while in healthcare and standardized solutions can dramatically improve the quality of healthcare delivered. There are some similarities to the approach taken in healthcare to efforts in other industries. The HL7 standards are developed with a healthcare model as its core foundation.

In the non-healthcare manufacturing world, the Open System Architecture for Computer Integrated Manufacturing (CIMOSA) has an excellent model driven approach to dealing with integration ${ }^{81}$. CIMOSA effort dates back to late eighties and continues to date with a single minded focus on modeling enterprise systems and deriving integration strategies from enterprise models.

The adoption of SOA has been slow in healthcare. It appears that the climate for that is changing and can lead to true integration and interoperability. The functional EHR model and the DSTU gives hope to that belief.

\section{REFERENCES}

Andrew Hinchley (2003). Understanding Version 3. A primer on the HL7 Version 3 Communication Standard, Alexander Moench Publishing, ISBN 3-933819-18-0, Munich, Germany.

HDTF (2004) Object Management Group, Health Domain Task Force (OMG HDTF, formerly CORBAmed). http:/healthcare.omg.org/Healthcare_info.htm (accessed 2004)

V. Jagannathan, K. Wreder, R. Glicksman, Y. Alsafadi (1998). "Objects in Healthcare - focus on standards," ACM Standards View, Summer 98.

AgentCities (2002) - Working group on Healthcare Applications. http://wwwcms.brookes.ac.uk/hcwg/LivDoc.pdf (accessed 2004)

CCOW (2004) HL7 Context Management Standar, Clinical Context Object Workgroup, http://www.h17.org/special/Committees/ccow_sigvi.htm (accessed 2004)

\footnotetext{
${ }^{81}$ Computer Integrated Manufacturing - Open System Architecture (CIMOSA). http://cimosa.de
} 
DICOM (2004) Digital Imaging and Communication in Medicine (DICOM), National Electrical Manufacturers Association, Rosslyn, VA (http://medical.nema.org/)

Q. Li, P. Shialane, N. F. Noy, M. A. Musen. (2000) "Ontology Acquisition from OnLine Knowledge Sources. AMIA Annual Symposium, Los Angeles. (http://smiweb.stanford.edu/pubs/SMI_Abstracts/SMI-2000-0850.html )

C. J. Buck (2002) "Step-by-step Medical Coding," W. B. Saunders Company, An Imprint of Elsevier Science, Philadelphia, PA, ISBN: 0-7216-9333-4.

UMLS (2004) Unified Medical Language System, National Library of Medicine, National Institutes of Health, USA (http://www.nlm.nih.gov/research/ umls/archive/2004AC/umlsdoc_2004ac.pdf, accessed December 2004)

SAML (2004) Secure Access Markup Language (SAML), Organization for the Advancement of Structured Information Standards (OASIS), Billerica, MA (http://www.oasis-open.org/committees/tc_home.php?wg_abbrev=security)

CDA (2004) Clinical Document Architecture (CDA), Health Level Seven, Inc. http://www.hl7.org/special/Committees/structure/struc.htm

ebXML (2001) Electronic Business XML (ebXML), UN/CEFACT and Organization for the Advancement of Structured Information Standards (OASIS), Billerica, MA http://www.oasisopen.org/committees/tc_home.php?wg_abbrev=ebxml-bp

Kotok, D.R.R.Webber (2002) ebXML: The New global standard for doing business over the internet. New Riders Publishing. ISBN: 0-7357-1117-8.

Institute of Medicine (2001) To Err is Human. Building a safer health system. ISBN\# 0-309-06837-1. The National Academy Press, 2001.

Institute of Medicine (2001b) Crossing the Quality Chasm: A new health system for the 21 st Century. ISBN\# 0-309-07280-8. The National Academy Press, 2001.

Institute of Medicine (2003) Committee on Data Standards for Patient Safety: Key Capabilities of an Electronic Health Record System: Letter Report. Washington, D.C., The National Academy Press, 2003.

HL7 (2004) EHR System Functional Model: A Major Development Towards Consensus on Electronic Health Record System Functionality. Available from www.h17.org. 